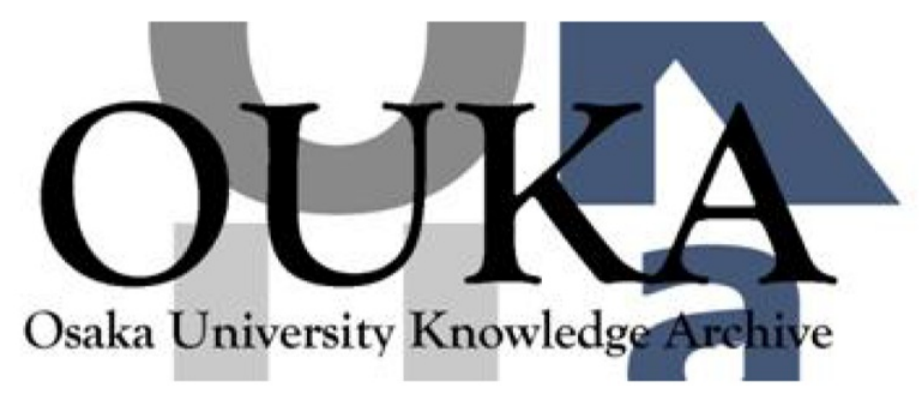

\begin{tabular}{|c|l|}
\hline Title & $\begin{array}{l}\text { Cryogenic deuterium target experiments with the } \\
\text { GEKKO XII, green laser system }\end{array}$ \\
\hline Author(s) & $\begin{array}{l}\text { Tanaka, Kazuo A.; Yamanaka, Tatsuhiko; } \\
\text { Nishihara, Katsunobu et al. }\end{array}$ \\
\hline Citation & Physics of Plasmas. 2(6) p. 2495-p. 2503 \\
\hline Issue Date & $1995-06$ \\
\hline oaire:version VoR \\
\hline URL & https://hdl. handle. net/11094/3377 \\
\hline rights & \\
\hline Note & \\
\hline
\end{tabular}

Osaka University Knowledge Archive : OUKA

https://ir. Library. osaka-u. ac. jp/

Osaka University 


\title{
Cryogenic deuterium target experiments with the GEKKO XII, green laser system*
}

\author{
Kazuo A. Tanaka, ${ }^{\dagger}$ Tatsuhiko Yamanaka, Katsunobu Nishihara, Takayoshi Norimatsu, \\ Noriaki Miyanaga, Hiroyuki Shiraga, Mitsuo Nakai, Yoneyoshi Kitagawa, Ryosuke Kodama, \\ Tadashi Kanabe, ,) Hiroshi Azechi, Manabu Heya, Takahisa Jitsuno, Masataka Kado, \\ Kunioki Mima, Masahiro Nakatsuka, Akio Nishiguchi, Hideaki Takabe, Masaru Takagi, \\ Kouji Tsubakimoto, Masahiro Tsukamoto, Yoshiaki Kato, Yasukazu Izawa, and \\ Sadao Nakaja) \\ Institute of Laser Enginèering, Osaka University, Suita, Osaka 565 Japan
}

(Received 14. November 1994; accepted 6 March 1995)

\begin{abstract}
A series of experiments were conducted using cryogenic deuterium targets to study fundamental physics and implosion dynamics with the GEKKO XII glass laser system [IEEE J. Quantum Electron. QE-17, 1639 (1981)]. Preheat sources were found to be due to a shock wave and hot electrons. A new method to measure the fuel $\rho R$ using proton spectra was employed. Measured in detail were the implosion dynamics of cryogenic deuterium foam with a plastic ablator and a $\mathrm{CH}$ shell with a controlled pressure of deuterium gas targets. Under current experimental conditions sources of nonuniformity were discussed in terms of Rayleigh-Taylor instability. (C) 1995 American Institute of Physics.
\end{abstract}

\section{INTRODUCTION}

Laser inertial confinement fusion ignition or reactor level target implosion typically requires the convergence rations $R_{i} / R_{f}\left(R_{i}\right.$ : the initial outer radius of the target and $R_{f}$ is the final compressed radius of the fuel) to be 20-40, final compressed fuel densities of the order of 1000 times the deuterium-tritium (D-T) liquid density, density radius product of $2-3 \mathrm{~g} / \mathrm{cm}^{2}$, and several $\mathrm{keV}$ hot spark temperature. Since a candidate for these targets may have a thick D-T layer and plastic ablator, ${ }^{1}$ it is necessary to develop the target fabrication and handling technique. By isentropically compressing the fuel of the ignition or high gain targets to a nearly degenerate state, the required laser energy is minimized. Any preheat in the fuel during the implosion alters this scenario and increases the required laser energy. ${ }^{2}$ On the other hand, the preheat might help reduce the growth of Rayleigh-Taylor (RT) instability. ${ }^{3}$ Thus the detailed preheat database should be essential for the ignition target design. Implosion experiments at the laser wavelengths at 527 and $351 \mathrm{~nm}$ indicated that the compressed density could be reached as high as 200-600 times the liquid fuel density. ${ }^{4}$ The corresponding neutron yields have been observed to be less than the simulation prediction, which have been attributed to fluid instabilities such as Rayleigh-Taylor instability. It is not clear what the details of the final phase of the compressions are in experiments, since the final compression phase happens within a short time $(<100 \mathrm{ps})$ and small spatial $(<50 \mu \mathrm{m})$ scales under current experimental conditions. This has been a limitation to the diagnostics. It is of critical importance to conduct implosion experiments using a cryogenic system to control deuterium fuel $\left(\mathrm{D}_{2}\right)$, pressure for studying (1) the fundamental physics and implosion performances of a thick fuel layer ( $>10 \mu \mathrm{m})$ with a plastic ablator,

\footnotetext{
*Paper 9IA1, Bull. Am. Phys. Soc. 39, 1750 (1994).

${ }^{\dagger}$ Invited speaker.

a) Also at the Faculty of Engineering, Osaka University, Suita 565 Japan.
}

a candidate for ignition and high gain experiments, and (2) details of the final phase of the compression to reveal the effect of fluid instability, shock wave, and preheat.

We have conducted a series of experiments using cryogenic systems in situ at the target vacuum chamber using a $527 \mathrm{~nm}$ laser wavelength with random phase plates ${ }^{5}$ to study these issues. In planer target experiments a cryogenic deuterium foam (CDF) plate was irradiated by one or two green laser beams. At a $3 \times 10^{14} . \mathrm{W} / \mathrm{cm}^{2}$ laser intensity, the major nonlinear instability in the corona region is two plasmon decay (TPD) instability. The temporally resolved observation of the blackbody emission on the rear side of planar CDF target shows the effect of hot electrons and shock heating. Proton spectra were shown to have a shift corresponding to the fuel $\rho R$ in spherical implosion experiments of CDF with a plastic ablator (CDFP) target. Implosion performance of CDFP targets shows that the implosion stays in one dimension until the shock wave released from the imploding shell wall collides at the target center and hits the shell again. Detailed x-ray measurements show that the core temperature is of the order of $1 \mathrm{keV}$ at the initial phase of the compression and then become cold within a 50 ps time scale. CD shells containing several tens of atmospheres of deuterium gas have been imploded to study the details of the compression. A new method that has been employed is MIXS (multiimaging x-ray streak camera), which is capable of taking 18 10 ps time frame images with a 10 ps time interval. For $40-50$ atm $\mathrm{D}_{2}$ pressure, the compressed cores show the effect of low modes $(l=4-6)$ by changing the laser focusing condition. D-D neutron yield was $0.5-1 \times 10^{8}$, which corresponds to the simulated yield slightly $(50-100 \mathrm{ps})$ after the convergence at the target center and then the reflected shock hitting the imploding shell for the first time. We then discuss possible modes in Rayleigh-Taylor instability for causing such a degradation of implosion performance. 


\section{EXPERIMENTAL CONDITIONS}

For fundamental physics studies planer targets were irradiated by several beams of the GEKKO XII glass laser system. ${ }^{6}$ Two beams of $527 \mathrm{~nm}$ laser light were focused on planar cryogenic deuterium foam (CDF) targets with an $f / 3$ lens through random phase plates (RPP) for the shock and corona instability experiments. The typical laser intensity was $3.4 \times 10^{14} \mathrm{~W} / \mathrm{cm}^{2}$ with a $600 \times 700 \mu \mathrm{m}$ oval focal spot and $1 \mathrm{~ns}$ at the full width at half-maximum (FWHM) pulse. The average density of CDF targets was $0.21 \mathrm{~g} / \mathrm{cm}^{3}$ with solid deuterium and polystyrene $\left[(\mathrm{C} 8 \mathrm{H} 8)_{n}\right]$ foam. CDF targets were coated with sodium carboxyl-methyl-cellulose (CMC, $Z=4.2$ ) for comparing the effect of preheat.

When a shock wave breaks out of a plane target, the rear side of the target radiate a blackbody emission, depending on the temperature behind the shock. The rear side of the CDF target was observed by the combination of a Schwarzschild microscope and an S-20 streak camera with a $2 \mu \mathrm{m}$ spatial resolution at the spectral region from 250 to $480 \mathrm{~nm}$. The system was calibrated to give a brightness temperature of a blackbody emission. The hard $x$-ray spectra from hot electron bremsstrahlung emissions in a $8.5-90 \mathrm{keV}$ photon range and time history of $20 \mathrm{keV} \times$ rays were monitored by a filter fluorescer X-ray detector and an X-ray streak camera. Stimulated Raman scattered (SRS) light was measured with the combination of a spectrometer and an S-1 streak camera. The three-half-harmonic (351 nm) light was also temporally resolved with a proper set of filters as a monitor of two plasmon decay instability. ${ }^{7}$

The neutron flight velocity is measured by a conventional time-of-flight method. Neutrons were measured by two scintillator-photomultiplier pairs. The secondary neutrons (due to the secondary $\mathrm{D}-\mathrm{T}$ reaction) were measured by two scintillator-photomultiplier (BC422) pairs. A proton spectrometer using a ceramic dipole magnet $(B=7 \mathrm{kG})$ measured the primary proton spectrum. The entrance slit of the spectrometer was $0.5 \times 2 \mathrm{~mm}$, located at $15.3 \mathrm{~cm}$ from the target. CR 39 plate was used for the detector of $2 \times 40 \mathrm{~mm}$ size, covering the energy range from 1 to $3.5 \mathrm{MeV}$.

Implosion experiments were conducted using the 12 beams of the GEKKO XII Nd:glass laser system at $527 \mathrm{~nm}$ with energies from 4 to $12 \mathrm{~kJ}$ with a pulse shape of a Gaussian or stacked Gaussian between 1.5 and $2.5 \mathrm{~ns}$ pulsewidth at half-maximum. The typical energy balance among the beams were within $5 \%$. For the recent implosion experiments the power balance of the 12 beams were controlled to within $5 \%$. The laser light was focused via an $f / 3$ lens with the RPP's. The focusing point was typically beyond the target by $D / R$ $=5$, where $D$ is the distance between the target center and the best focus and $R$ is the spherical target radius. Estimated overall irradiation nonuniformity was less than $20 \%$ root mean square (RMS). Targets were either cryogenic deuterium foam with a plastic ablator (CDFP) or $\mathrm{CH}$ shell with 50 atm $D_{2}$ gas. Typical sizes of the CDFP are $4 \mu \mathrm{m}$ ablator, 10 $\mu \mathrm{m}$ foam and deuterium layer, and $500-700 \mu \mathrm{m}$ diameter. $\mathrm{CH}$ shells of $500 \mu \mathrm{m}$ diameter have $7 \mu \mathrm{m}$ thick walls with $50 \mathrm{~atm}$ deuterium gas. A new method utilizing an $\mathrm{x}$-ray streak camera with multiple pinholes (multi-imaging $x$-ray streak camera: MIXS) and computer processing of data has
$10 \mu \mathrm{m}$

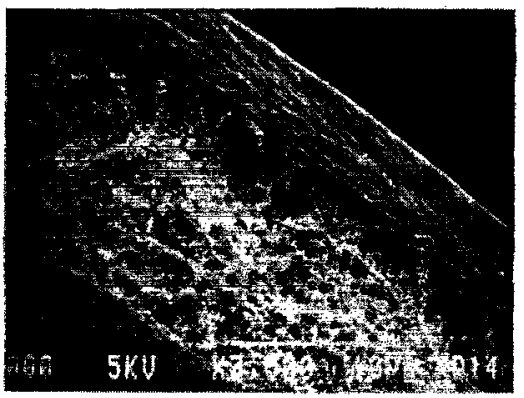

FIG. 1. A cross section of foam with plastic ablator. The size of the foam structures is less than $200 \mathrm{~nm}$.

been introduced to have 10 ps time frames for the twodimensional (2-D) images of imploded cores with a 10 ps interval. ${ }^{8}$ A conventional $x$-ray streak camera with three pinholes was used to estimate the temperature of the core using different $x$-ray filters.

\section{FOAM SHELL FABRICATION AND TARGET HANDLING}

In earlier plane geometry experiments, foam targets were made with a freeze dry method. A 2.5 wt.\% 1,4-dioxane solution of polystyrene (PS) in a planar mandrel was quickly frozen by dipping it in liquid nitrogen to induce phase separation of the dioxane and PS. The frozen solution was placed in a vacuum chamber to leave a PS foam plate. The cell size and the density of the foam fabricated by this method were 4 $\mu \mathrm{m}$ and $40 \mathrm{mg} / \mathrm{cc}$. Recently, foam shells have been fabricated with a density-matched emulsion method and droplet generator in a liquid phase. ${ }^{10}$ Trimethylpropane trimethacrylate (TMPT) was used as a foam network former in the oil phase. In the water the oil phase with $3 \mathrm{wt} . \%$ TMPT was mixed to make a $W$ (water)/O (oil) emulsion. Then the $W / O$ emulsion was again poured into a second water phase to make a $\mathrm{W} / \mathrm{O} / \mathrm{W}$ emulsion. This emulsion was heated at $70^{\circ} \mathrm{C}$ to polymerize in the oil phase. After the polymerization, the shells were dried to obtain foam shells. In order to produce foam shells with controlled diameter and wall thickness, a dual nozzle in an orifice droplet generator in the liquid phase was used. Foam shells were plastic coated by a newly developed interfacial polycondensation technique. ${ }^{11}$ This technique using hydroxyethyl-cellulose or poly-vinyl phenol had an excellent spherical uniformity as good as $98 \%$. A cross section of a foam ( $10 \mu \mathrm{m}$ thickness) shell with a $4 \mu \mathrm{m}$ plastic ablator is shown in Fig. 1, where one can see that the cell size of the foam structure is less than $1 \mu \mathrm{m}$ for $50 \mathrm{mg} / \mathrm{cm}^{3}$ density. A power spectrum of the surface of a plastic ablator is shown in Fig. 2, where the surface roughness is $3 \mathrm{~nm}$ RMS.

Two cryostats were used in these series of experiments. For the earlier planer experiments, a cryostat ${ }^{12}$ containing a quartz pot to reserve liquid $D_{2}$ was installed in the target chamber of the GEKKO XII laser system. The plane foam suspended on a glass stalk was immersed into the liquid deuterium to one-third of the whole height. Since the foam could be wet with liquid deuterium, the foam plane was satu- 


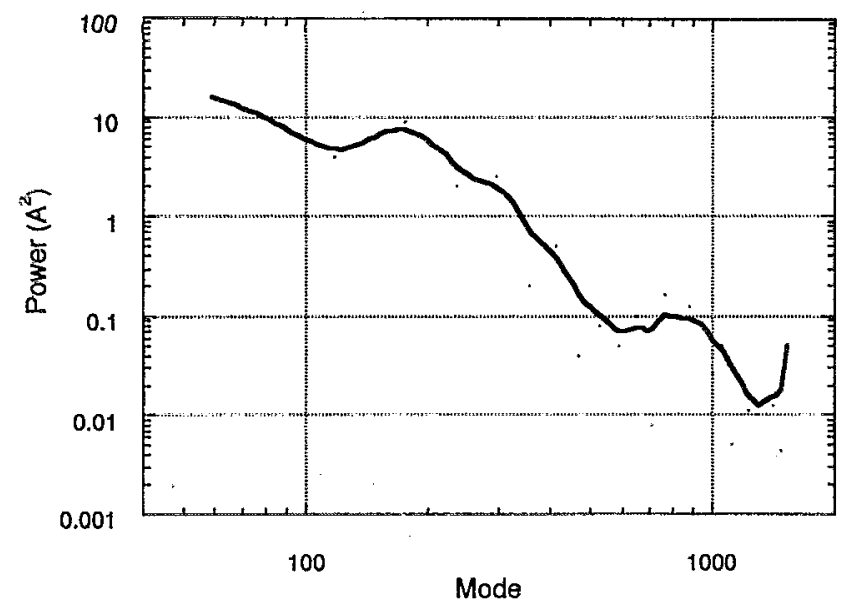

FIG. 2. A power spectrum of the surface of a plastic ablator coated on a spherical foam shell. The surface roughness is $3 \mathrm{~nm}$ RMS.

rated with liquid deuterium. After locating the foam plane at the firing position, the whole target was frozen. The target was exposed in vacuum for the laser irradiation by reducing the deuterium gas around the target and simultaneously increasing the cooling rate of the cryostat. The cryogenic shroud was retracted $20 \mathrm{~ms}$ prior to the laser irradiation. The sublimation of solid deuterium before the laser irradiation was estimated to be less than $2 \mu \mathrm{m}$ in thickness. The sublimation rate, which depends on both time history of the surrounding vapor pressure and temperature of the target, was determined experimentally by measuring the mass of the solid deuterium in a foam shell in a similar circumstance. ${ }^{13}$

A cryostat equipped with a high pressure fill chamber ${ }^{14}$ was employed for implosion experiments of spherical foam shells with a plastic ablator. A foam shell with a plastic ablator was held with a $2 \mu \mathrm{m}$ diameter polyester fiber attached to a glass stalk. The shell was pressurized with $100-130 \mathrm{~atm}$ deuterium gas in a cryostat at the vacuum target chamber. The fill pressure was determined so that the foam layer was slightly overfilled with $\mathrm{D}_{2}$ at the temperature when it was fired. Then the cryostat was cooled down to form a liquid layer of $\mathrm{D}_{2}$ in the foam structure at below $35 \mathrm{~K}$. A schematic of the cryostat system is shown in Fig. 3. A upper pylon is to position the target at the laser focusing and a lower pylon is to fuel and cool the target. The lower cryostat consists of a gas-filling chamber and a cryogenic shroud retractable independently for the target shooting. An indium ring was used to seal the high pressure deuterium gas between the filling chamber and the target positioner. The filling rate was controlled within a quarter of the collapsing pressure so as not to break the target shell. A typical filling rate was $80 \mathrm{~min}$ for a $600 \mu \mathrm{m}$ diameter foam shell with a $4 \mu \mathrm{m}$ plastic ablator. After lowering the cryostat temperature to $40 \mathrm{~K}$, the residual $D_{2}$ gas was evacuated from the filling chamber. The typical time to bring the target ready for the laser shot is $2 \mathrm{~h}$ after the $D_{2}$ filling. After the shell was cooled to the desired temperature, the uniformity of the $\mathrm{D}_{2}$ layer was monitored with interference microscopes in situ. The shroud was retracted 20 $\mathrm{ms}$ before the laser irradiation. Estimated temperature rise on
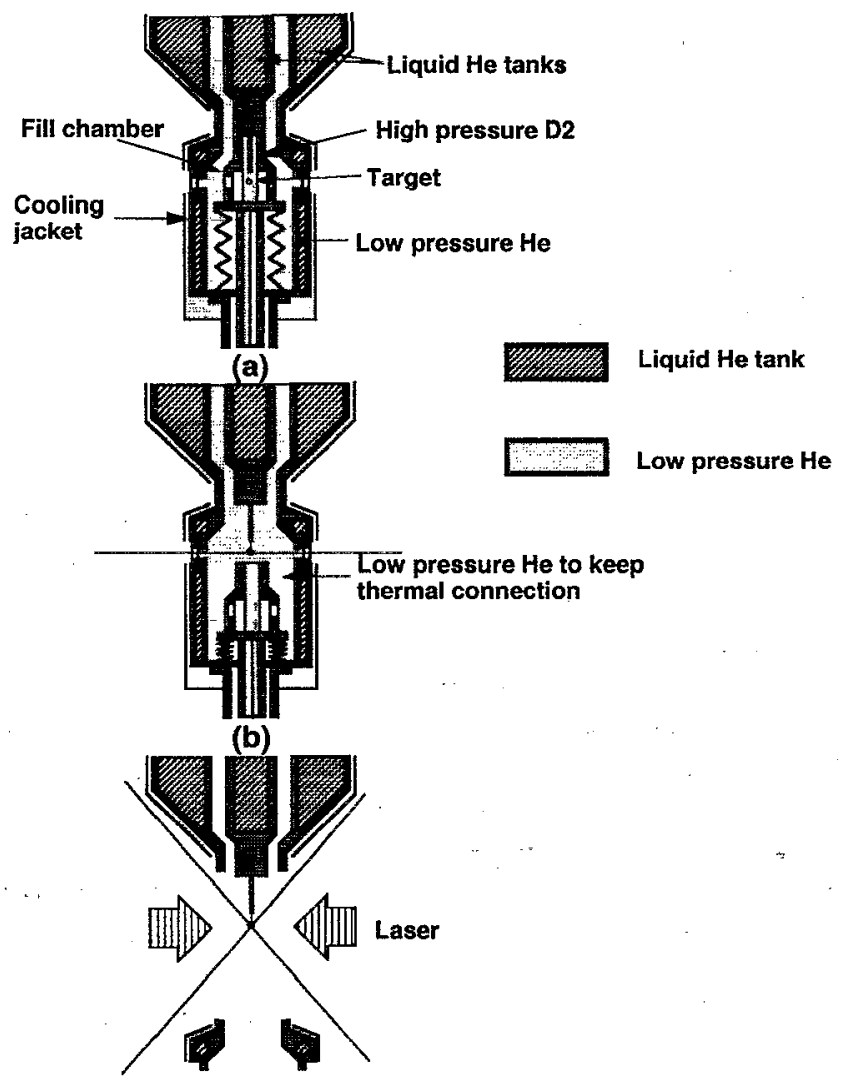

(c)

FIG. 3. A schematic of the cryostat system used for the implosion experiment. (a) Filling mode, (b) target alignment, and (c) laser irradiation.

the target was less than $1.4 \mathrm{~K}$ from ambient blackbody radiation.

\section{SHOCK AND HOT ELECTRON PREHEAT}

As mentioned in Sec. II Experimental conditions, the shock wave and other preheat were monitored by the rear side emission of planer targets. The front side of the CDF target was irradiated at $3.4 \times 10^{14} \mathrm{~W} / \mathrm{cm}^{2}$ and the rear side emission due to preheats is measured. Figure 4 shows that the rear side emissions were composed of mainly two components: a sharp rising shock wave and nonlocal heating due to hot electrons. The figure indicates that the shock wave

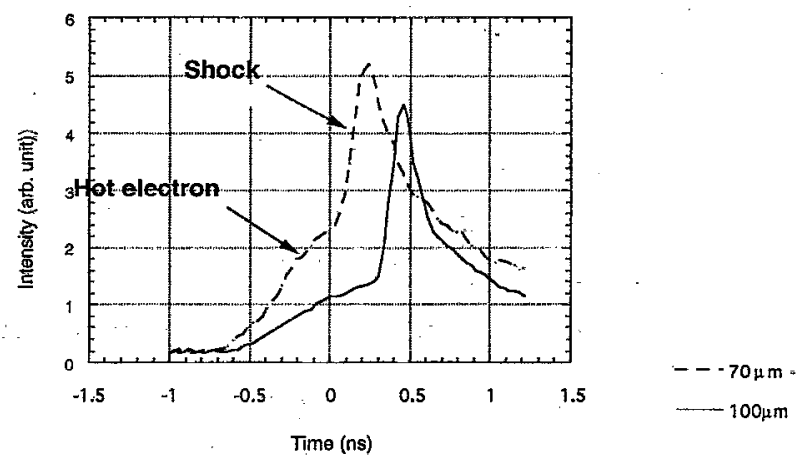

FIG. 4. Time-resulved rear emission of CDF target irradiated at $3.4 \times 10^{14}$ W/ $\mathrm{cm}^{2}$. The target thickness were 68 and $100 \mu \mathrm{m}$. 


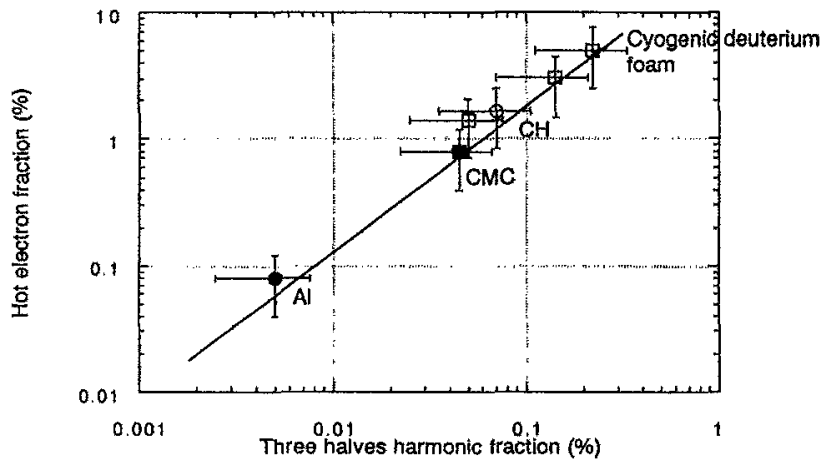

FIG. 5. Temporal shapes of SRS three-half harmonic, and $20 \mathrm{keV} x$ rays.

component is delayed with increasing the target thickness from $68 \mu \mathrm{m}$ to $100 \mu \mathrm{m}$, but the intensity did not change much. The peak temperature of the shock was estimated to be $35-40 \mathrm{eV}$ based on the assumption that the emission is a blackbody. This brightness temperature was calibrated using an aluminum target shot with the known SESAME database. The other component in front of the shock wave signal did not show such delay as the one seen in the shock signals and the intensity was reduced with increasing the target thickness. It is estimated that this level of heating would be possible with hot electrons with about $15 \mathrm{keV}$ temperature. In this experiment heating due to $x$ rays has been excluded because of the low atomic number of the CDF target. One of the sources of hot electrons was identified due to TPD instability at the quarter-critical plasma density as shown in Fig. 5. TPD is a nonlinear parametric instability, where the incident laser photon decays into two electron plasma waves at the quarter-critical plasma density. The generated plasma waves could create hot electrons corresponding to the phase velocity of the plasma waves. Figure 5 shows the convective SRS, three-half harmonic due to TPD, and $20 \mathrm{keV}$ hard $\mathrm{x}$-ray temporal histories. The similarity between the three-half harmonic and $20 \mathrm{keV}$ hard $\mathrm{x}$ rays indicates that the hard $\mathrm{x}$-ray component is related to the TPD instability, not to the convective SRS. Actually in this experiment the level of SRS was reduced by more than $10^{2}$ with the use of RPP's, while the level of three-half harmonic as a monitor of TPD was not affected by the RPP's. This result is consistent with the ones in Ref. 15. The three-half-harmonic intensity was well correlated with hot electron fraction as shown in Fig. 6. The data were taken for $\mathrm{CDF}$, plastic $(\mathrm{CH})$, sodium carboxylmethyl-cellulose (CMC), and aluminum (Al) planar targets at laser intensities between 1.8 and $3.4 \times 10^{14} \mathrm{~W} / \mathrm{cm}^{2}$, indicating that the TPD fraction decreases with increasing the atomic number. The hot electron contents were estimated from the hard $x$-ray time-integrated spectral measurement, whose observation range was from $8.5 \mathrm{keV}$ to $90 \mathrm{keV} .{ }^{16}$ The spectra above $20 \mathrm{keV}$ photon energy were well represented with a Maxwellian distribution. The fraction of hot electrons to the incident laser energy was about $4 \% \pm 2 \%$ with a hot electron temperature of $15 \mathrm{keV}$ for the CDF target at $3.4 \times 10^{14}$ $\mathrm{W} / \mathrm{cm}^{2}$. This fraction was reduced by a factor of 5 for the CMC targets. We could obtain the best fit of the experimentally observed emission due to the hot electron and shock

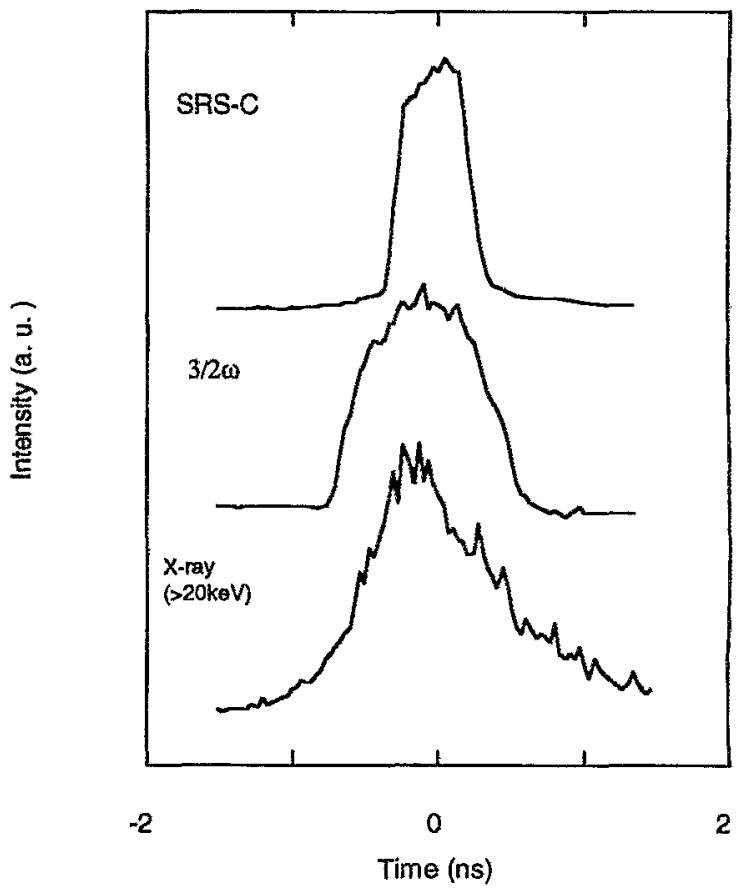

FIG. 6. Hot electron fraction versus three harmonic intensity. Hot electron is well correlated with three-half-harmonic intensity (two plasmon decay instability).

wave with our Fokker-Planck code, HIMICO, ${ }^{17}$ simulation, corresponding to $4 \%$ of the incident laser energy converted to $14 \mathrm{keV}$ temperature and the thermal tail electrons, consistent with the experimentally observed values. The fitting was shown in Fig. 7, where the width shown in the simulation is due to the numbers read at the first and second layer of the simulation.

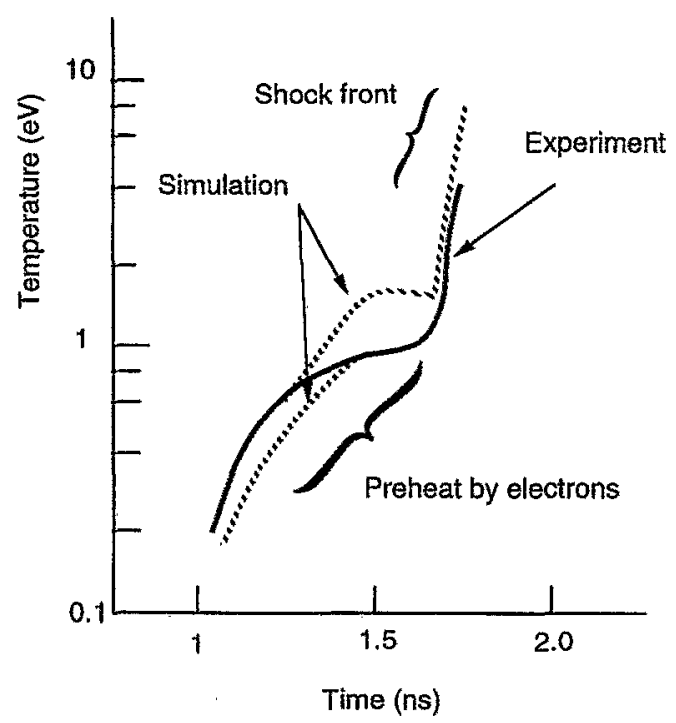

FIG. 7. Fitting by Fokker-Planck code simulation to the observed hot electron heating. This simulation fit corresponds to $4 \%$ of the incident energy converted to $14 \mathrm{keV}$ hot electrons and thermal tail electrons. 

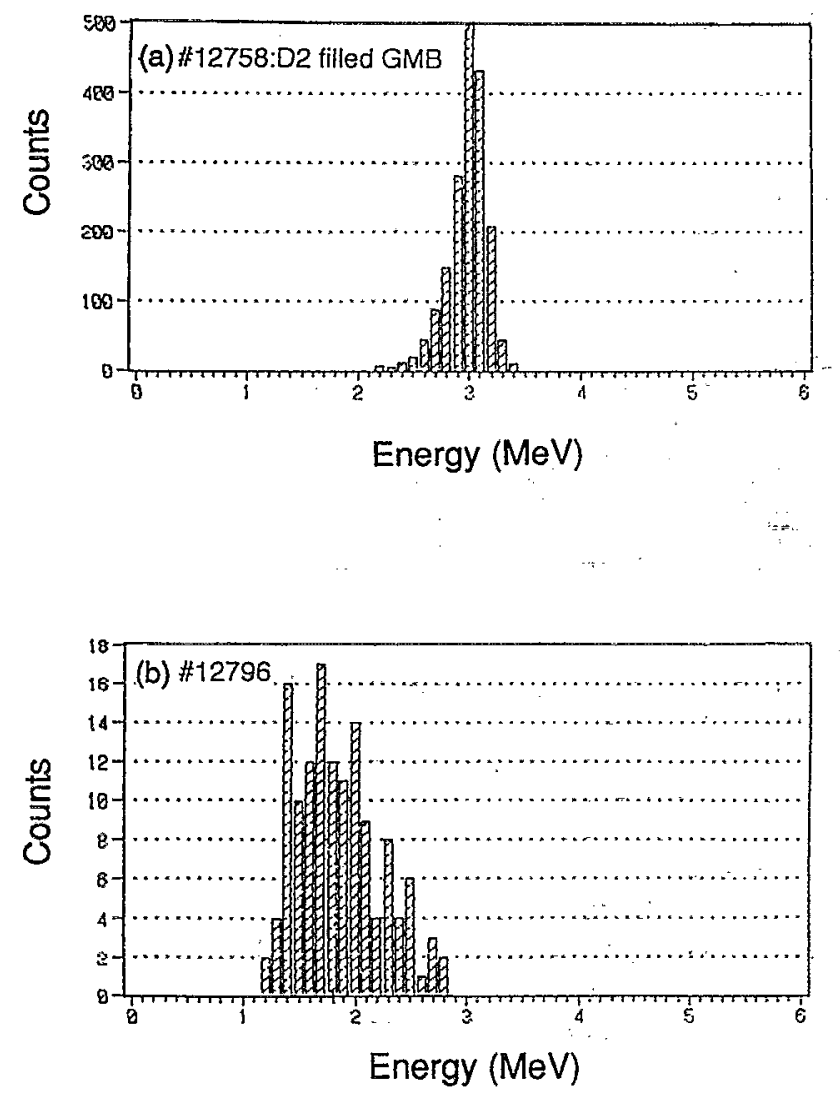

FIG. 8. Proton spectra for (a) glass microballoon shell and (b) CDFP target. Low density implosions associated with GMB show almost no shift, while the CDFP implosion shows a clear shift from its original position.

\section{FUSION PRODUCT}

As a new and alternative way to measure the fuel $\rho R$ to a secondary reaction method, ${ }^{18}$ proton spectra were measured. $3 \mathrm{MeV}$ protons produced in the D-D fusion reaction go through the surrounding high density fuel layer and are detected by the proton spectrometer. The proton spectrum is shifted because of the energy loss of the compressed fuel layer. Advantages of this method over the secondary method are that the absolute count of neutrons is not necessary for the measurement and $\gamma$-ray noise problem at the secondary neutron method could be avoided. In the secondary neutron method $\gamma$ rays generated at the diagnostics by both primary and secondary neutrons often obscure the time-resolved signals. ${ }^{18}$ Figures $8(a)$ and $8(b)$ show proton spectra from a simple $\mathrm{D}_{2}$ filled (11 atm) glass microballoon (GMB) target (621 $\mu \mathrm{m}$ diameter) and a CDF with a $4 \mu \mathrm{m}$ plastic (CDFP) ablator target (590 $\mu \mathrm{m}$ diameter). The foam layer thickness was $10 \mu \mathrm{m}$ filled with cryogenic $D_{2}$, which corresponds to $100 \mathrm{~atm} \mathrm{D}_{2}$ gas at room temperature. The laser intensity was $4 \times 10^{14} \mathrm{~W} / \mathrm{cm}^{2}$ for the GMB and the CDFP target with a 2 ns pulse duration with $8 \mathrm{~kJ}$ laser energies. For the CDFP shot, the deuterium temperature was $20 \mathrm{~K}$, where the $D_{2}$ layer was kept in a liquid phase. Comparing these two spectra, the spectrum from the GMB [Fig. 8(a)] shows little shift from its original position of $3 \mathrm{MeV}$, while the one from the CDFP target indicates the peak shift at around $1.8 \mathrm{MeV}$. The energy spread observed in Fig. 8(a) indicates the spectrometer reso-

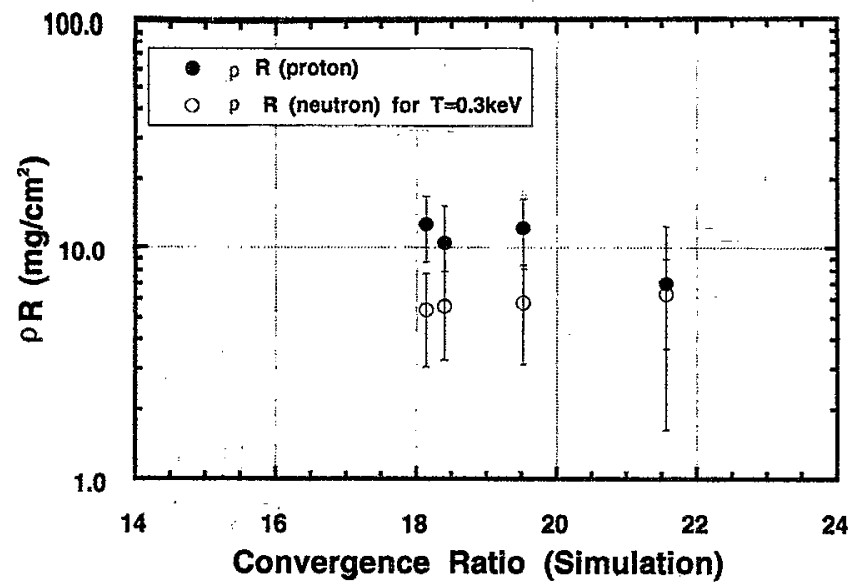

FIG. 9. $\rho R$ values versus calculated convergence ratio for CDFP targets. Also plotted are the ones estimated with the secondary neutron method. Multiplying 0.4 to the proton values to take account the plastic foam density, the fuel $\rho R$ values are in good agreement with the secondary neutron method.

lution limited by the Doppler broadening. According to the energy loss calculation including the Fermi degeneracy, ${ }^{19}$ a $1.2 \mathrm{MeV}$ peak shift corresponds to $\rho R$ about $12 \pm 4 \mathrm{mg} / \mathrm{cm}^{2}$ assuming the ion and electron temperatures are similar and to be $0.1-1.0 \mathrm{keV}$. The error originates from the spectral broadening. Here $0.3 \mathrm{keV}$ was assumed for the temperature since the main fuel layer temperature was $0.3 \mathrm{keV}$ in a onedimensional (1-D) hydrodynamic code simulation. The calculated $\rho R$ values are a function of the temperature within a factor 2 between 0.1 and $1 \mathrm{keV}$ and are indicative that the measurement is valid to $\rho R=40 \mathrm{mg} / \mathrm{cm}^{3}$ at $1 \mathrm{keV}$ temperature for solid or liquid $D_{2}$ density. The $\rho R$ values are plotted with the ones estimated with the secondary neutron scheme in Fig. 9. The difference seen in the figure between the proton and the secondary neutron methods is attributed to the additional stopping caused by foam plastic. Taking into account the foam density in the $\mathrm{D}_{2}$ and foam layer, the fuel $\rho R$ values should be 0.4 times the values in the graph for the proton measurement. Thus both methods are in good agreement. This indicates that the proton spectrum could be used as an alternative way to estimate the $\rho R$ values of compressed fuel.

\section{IMPLOSION EXPERIMENT}

Implosion experiments were conducted using the experimental conditions mentioned earlier. Two types of cryogenic targets were used: cryogenic deuterium foam with a plastic ablator (CDFP) and a $\mathrm{CH}$ shell with deuterium gas pressurized up to $50 \mathrm{~atm}$. A time history and MIXS images of the imploded cores are shown in Figs. 10(a) and 10(b) for \#15253 CDFP target shot. The observed photon energy was $1-2 \mathrm{keV}$, mainly from the bremsstrahlung emission of the fuel. The target diameter, plastic ablator thickness, and deuterium-in-foam layer thickness were $655 \mu \mathrm{m}, 4 \mu \mathrm{m}$, and $10 \mu \mathrm{m}$, respectively. The laser energy and intensity were 3.8 $\mathrm{kJ}$ in a $2.5 \mathrm{~ns}$ pulse (stacked Gaussian shape) and $7.3 \times 10^{13}$ $\mathrm{W} / \mathrm{cm}^{2}$. Measured D-D neutrons and fuel $\rho R$ were $7 \times 10^{6}$ and $8 \mathrm{mg} / \mathrm{cm}^{2}$. In Fig. 10(a), the temperature history of the 
(a)

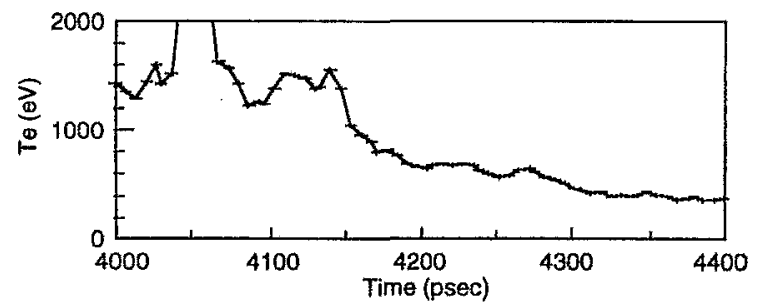

(b)

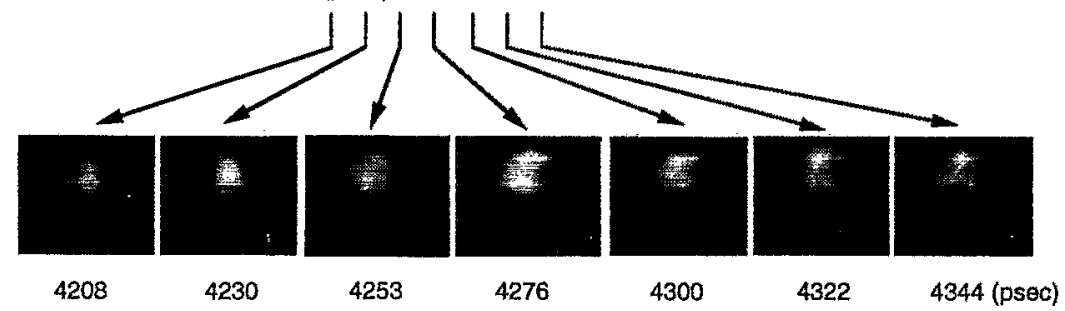

FIG. 10. (a) Temperature history and (b) MIXS images for CDFP targets. Time frame has about 10 ps opening with a 10 ps time interval. While low mode structures are seen, the core temperature drops suddenly.

core is plotted. The temperature has been deduced from another $\mathrm{X}$-ray streak camera measurement with the combination of multiple pinholes and different $x$-ray filters. The core 2-D images of Fig. 10(b) show low ( $l=4$ or so) mode distortions and the intensity increases until the $4276 \mathrm{ps}$ time frame. However, the temperature of the core starts decreasing at $4150 \mathrm{ps}$ and a sharp drop is seen until $4200 \mathrm{ps}$ from $1.5 \mathrm{keV}$ to $800 \mathrm{eV}$. This timing corresponds to the shock first bounce against the shell. ${ }^{20}$ After that time, the temperature decreases rather slowly to a $400 \mathrm{eV}$ level at the 4344 ps frame. Comparing these two figures, the intensity of the 2-D images still increases when the temperature starts decreasing. The observed photon in the 2-D image is above $1 \mathrm{keV}$. Since the temperature was deduced from $1 \mathrm{keV}$ and $2 \mathrm{keV}$ photon windows, the temperature decrease corresponds to the reduction of the $2 \mathrm{keV}$ window $\mathrm{x}$ rays. The core structures showing low modes do not change much between the frames, while the temperature is already reduced.

$\mathrm{CH}$ shells with pressurized deuterium fuel were also imploded to study further details of implosions, using the same cryogenic system to prevent the target from exploding before the laser shot. Typically the ambient cryogenic temperature around the target was $35 \mathrm{~K}$. Time histories of $\mathrm{x}$ rays from imploded cores were shown in Figs. 11(a) and 11(c) for the focusing conditions $D / R=4$ (\#15591) and 5 (\#15580). In these shots $x$ rays came mainly from 0.3 atm Ar seed gas mixed with the deuterium fuel. The target diameter, shell thickness, laser energy, and laser intensity were $486, \mu \mathrm{m}$ $7.23 \mu \mathrm{m}, 5.7 \mathrm{~kJ}$, and $5.7 \times 10^{14} \mathrm{~W} / \mathrm{cm}^{2}$ for $\# 15580$ and 503 $\mu \mathrm{m}, 6.87 \mu \mathrm{m}, 5.6 \mathrm{~kJ}, 5.2 \times 10^{14} \mathrm{~W} / \mathrm{cm}^{2}$ for \#15591. Deuterium pressure was $50 \mathrm{~atm}$ for both shots. In this experiment the power balance of 12 laser beams was controlled within $5 \%$ as well as the $5 \%$ energy balance. Observed neutrons were $5 \times 10^{7}$ for $\# 15580$ and $9.3 \times 10^{7}$ for $\# 15591$. Simultaneously the radius-time $(R-T)$ diagrams from HISHO 1-D simulations were attached for comparison in Figs. 11(b) and 11(d). The observed and the predicted time histories were shown as a line and hatched lines in Figs. 11(a) and 11(c). The observed line shows a relatively good fit until $2.31 \mathrm{~ns}$ compared to that predicted. After that time the predicted $x$-ray intensity increases rapidly, while the observed decreases. The simulated $R-T$ diagram at around the maximum compression shows the shock released into the deuterium fuel gas moves ahead of the imploding shell and collides at the target center. Then the shock wave starts oscillating between the imploding shell and the target center and heat the fuel and seed gas, resulting in the x-ray intensity increase. The timing of observed $x$-ray intensity decrease corresponds to when the shock comes into the target center for the second time in the simulation. This timing is also consistent with the simulated time of the observed neutron yield. Temporal features seen for $D / R=4$ in Fig. 11(a) was reproduced for the $D / R=5$ shot as shown in Fig. 11(c). The timing of the observed $x$-ray decrease also corresponds to the time for the second shock arrival to the target center in the simulation. The MIXS images at their maximum intensities were shown for $D / R=4$ and $D / R=5$ in Figs. 12(a) and 12(b). One notes that Fig. 12(a) of the core image shows somewhat a square feature, while Fig. 12(b) shows a more round symmetric core. It is important to point out that these detailed features were detected only with improved time resolutions made possible by the MIXS. Conventional x-ray framing cameras with an 80 ps time window showed no difference for the laser focusing condition such as the ones used here because of a time blurring effect even within the 80 ps time frame.

For CDFP and CH with 50 atm $\mathrm{D}_{2}$ gas target shots, core structures were seen in the MIXS images and the neutron and $\rho R$ values were not as high as the ones predicted by the HISHO simulation. If these are due to nonuniformities of laser irradiation and/or target structures and subsequent Rayleigh-Taylor instability, it is important to discuss what kind of nonuniformities could be responsible. Sources of nonuniformities could be the surface roughness of plastic ablator (20 nm RMS for a CDFP shell and 4 nm RMS for a $\mathrm{CH}$ shell), the density deviations in the foam and deuterium fuel layer (typical foam cell size is of the order of $200 \mathrm{~nm}$ ), the laser beam power balance, the finite beam number effect, 
(a)

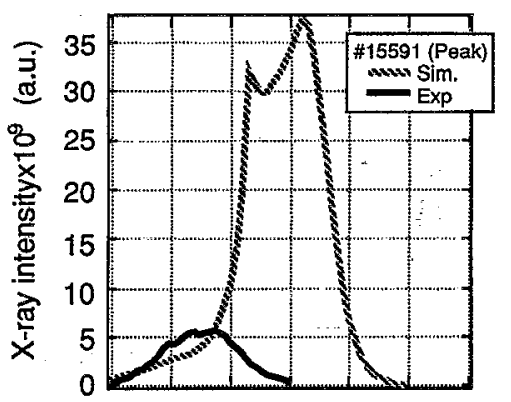

(b)

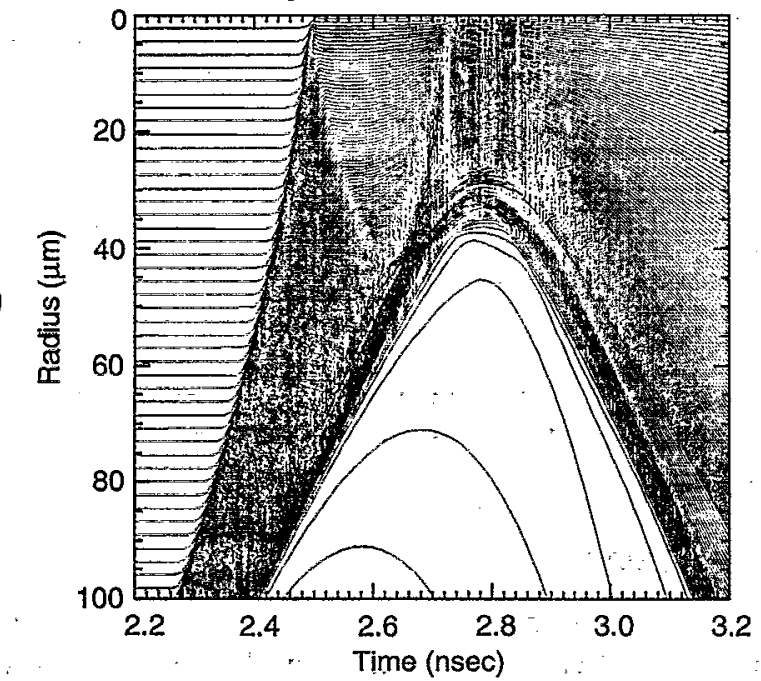

(c)

(d)

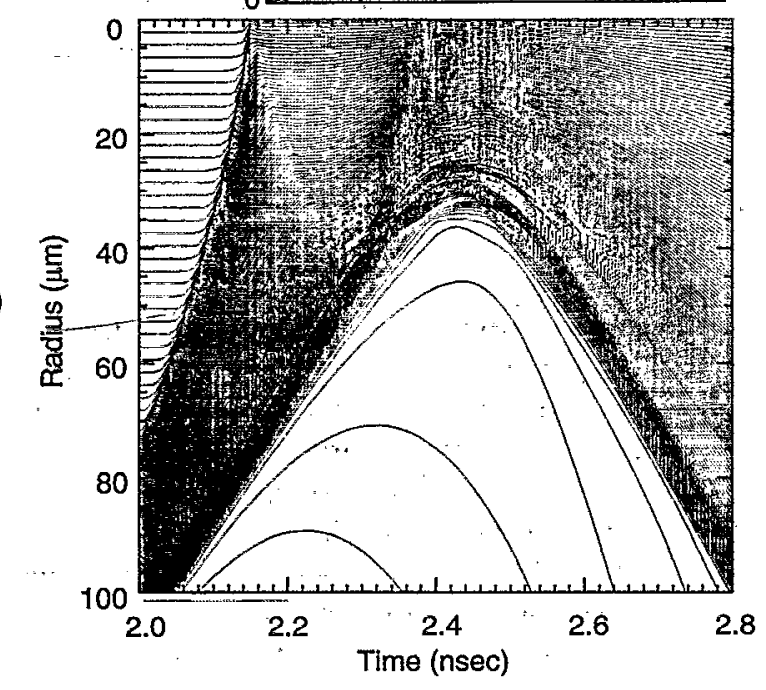

FIG. 11. (a) Observed and predicted $\mathrm{x}$-ray intensity time histories, (b) flow diagram from 1-D HISHO simulation for $\# 15591$ with $D / R=4$. (c) Observed and predicted $\mathrm{X}$-ray intensity time histories, (d) flow diägram from 1-D HISHO simulation for $\# 15580$ with $D / R=5$. (a)

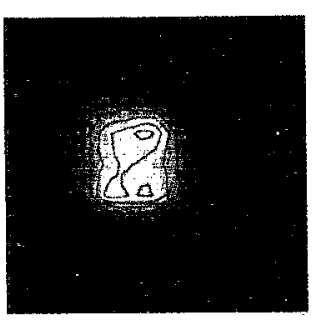

$D / R=4(t=2597 \mathrm{psec})$ (b)

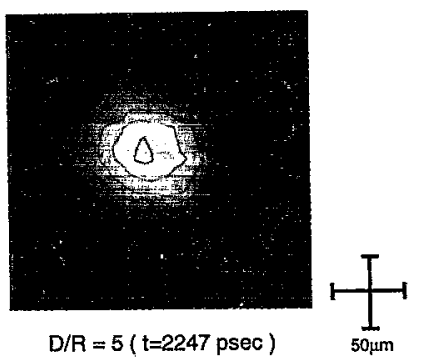

FIG. 12. MIXS images for (a) $D / R=4$ and (b) $D / R=5$. (b) $D / R=4$ shows low mode structures, which is consistent with the expected irradiation nonuniformity for mode 6.

and the structures in the beam spot (RPP typically creates a Gaussian-like focal spot pattern with many 5-6 $\mu \mathrm{m}$ sharp intensity spikes). On the other hand, the irradiation nonuniformities with very small wavelengths might be smoothed out by thermal electrons in plasmas as $\exp (-k D)$, where $k$ is the wave number $(2 \pi / \lambda)$ of the perturbation and $D$ is the separation distance between the ablation front and laser absorption region. ${ }^{21}$ For example, irradiation nonuniformity with a $5 \mu \mathrm{m}$ wavelength could be $1 / 100$ with a $5 \mu \mathrm{m}$ separation distance. Similarly the RT growth would be reduced by ablation stabilization for short wavelength nonuniformities as the growth rate, $\gamma=\alpha(k g)^{1 / 2}-\beta k v_{a}$, where $\alpha$ is the Atwood number, $\gamma$ is the acceleration, $\beta$ is an adjusting factor expected to be $3-4$, and $v_{a}$ is the ablation velocity. For a mode 100 and $g=10^{16} \mathrm{~cm} / \mathrm{s}^{2}$, the ablation velocity would be required to be close to $10^{6} \mathrm{~cm} / \mathrm{s}$ for the effective reduction of the RT growth rate.

In our calculations it was known that the $D / R=4$ and $D / R=5$ conditions create $22 \%$ and $14 \%$ nonuniformities for mode 6 on the irradiation target surface, indicating that there might be 1.6 times: improvement for this low mode for $D / R=5$ [Fig. 12(b)] than $D / R=4$ [Fig. 12(a)]. This improvement is reflected in the MIXS pictures in Figs. 12(a) and 12(b), but not in the x-ray temporal histories in Figs. 11(a) and 11(c). In other words the MIXS images certainly show the effect of improvement of the low mode illumination uniformity. However the $x$-ray intensity, which is a function of the core plasma temperature, is affected by the modes not observable in the MIXS pictures. Figure 13 shows RayleighTaylor growth results from HISHO simulation ${ }^{22}$ for the above two $\mathrm{CH}$ shell target shots. In the simulation a forced RT equation for the ablation surface distortions $\xi$ as $d^{2} \xi_{k} / d t^{2}=\gamma_{k}^{2} \xi_{k}+\delta g_{k}$, where $\delta g_{k}$ is the gravity perturbation and $\gamma_{k}$ is the RT growth rate for the wave number $k$, which is. related to the Legendre mode number $l$ and the instantaneous radius of the ablation front $R_{a}$ as $k R_{a}=[l(l+1)]^{1 / 2}$. For the RT growth, $\gamma_{k}$, the Takabe formula ${ }^{23}$ was used. Initial imprint was taken into account by using the quasistational deflagration model. ${ }^{24}$ The mode saturation form was taken from Ref. 25: For the thermal smoothing the cloudy day model was assumed. ${ }^{21}$ The growth was calculated until the shell acceleration was over. In the low mode area, the dominant modes are 2 and 6 for both target shots. Mode 2 comes from the measured laser spot pattern taken into account in the 


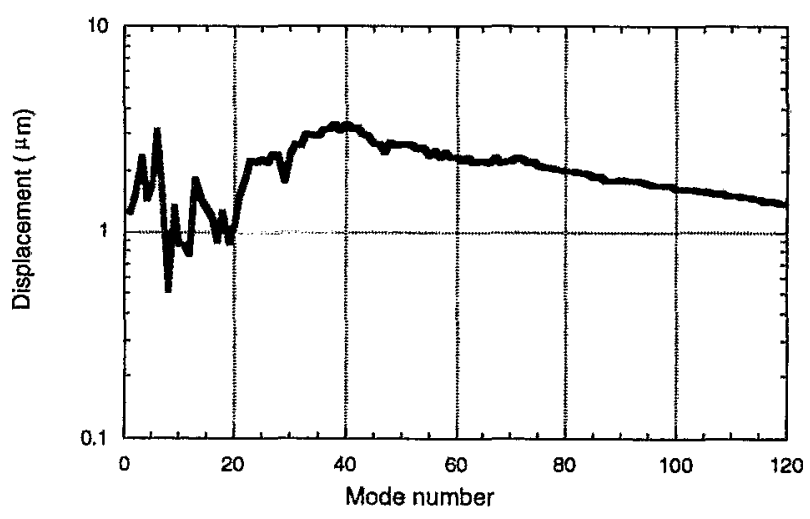

(a)

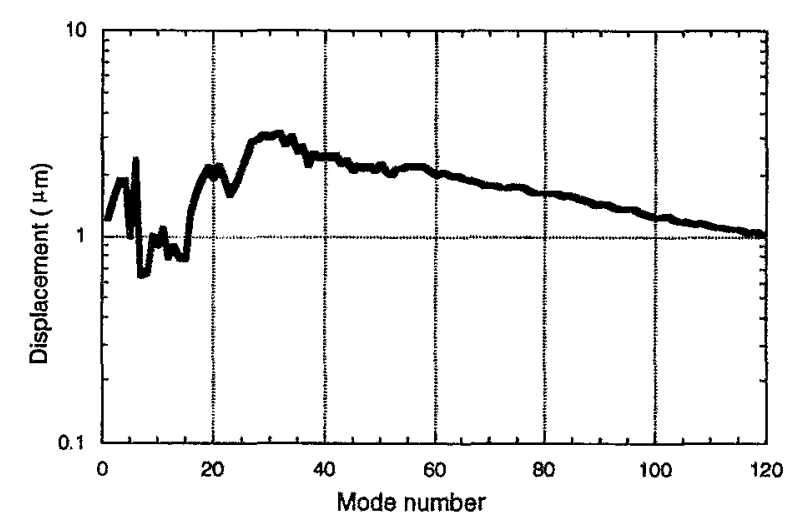

(b)

FIG. 13. Rayleigh-Taylor growth results for (a) \#15591 and (b) \#15580 shots. Two major mode areas are seen: one at low mode 6 and the other at modes between 30 and 40 .

simulation, while mode 6 comes from the focusing condition and the 12 beam effect. Figure 13(a) for $D / R=4$ actually shows that the displacement growth at mode 6 is large compared to Fig. $13(\mathrm{~b})$ the for $D / R=5$ case, consistent with Fig. 12 of the MIXS pictures. In the mid-mode range other major peaks at mode 30 and 40 are expected as shown in the Fig. 13. Considering the facts that during the implosion of the CDFP target the core temperature drops suddenly while the overall core shape in the MIXS did not change much with low modes, and the $\mathrm{CH}$ shell with gaseous $\mathrm{D}_{2}$ shots shows low mode structures according to the focusing conditions, but still shows similar performances for the neutron product (which is a function of core temperature) and $\mathrm{x}$-ray intensity histories, it might be reasonable to say that the implosion performances such as neutrons and temperature are affected not by the low modes, but rather modes not observable in the MIXS images such as the modes peaking between 20 and 50 as shown in Fig. 13. Using a spectrally dispersed amplified spontaneous emission (ASE) mode or partially coherent laser light in the laser system, ${ }^{26}$ it could be possible to reduce these modes drastically. For the same shot condition as the \#15580 [Fig. 11(d)] but employing the laser intensity distribution of an ASE beam, it is expected to reduce these mid to high modes by almost an order of magnitude as shown in Fig. 14. Only low modes resulting from the finite beam num-

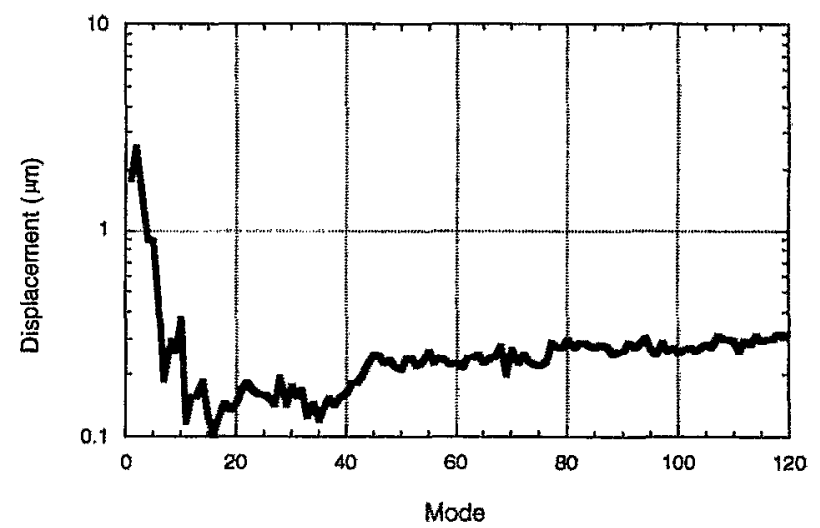

FIG. 14. For the condition of $\# 15580$ but the laser light. Spectrally dispersed amplified spontaneous emission (ASE) or a partially coherent light focused pattern was used. With improved mid to high mode illumination uniformity RT growth at these modes could be much reduced.

ber effect should remain for the shell displacements larger than $0.5 \mu \mathrm{m}$ with the uniformity improved by the ASE laser beams.

\section{SUMMARY}

Using a cryogenic system in situ at the target chamber a number of different experiments have been conducted. Target fabrication techniques and surface roughness data were summarized.

Preheat was studied using planar CDF targets, measuring scattered light, hard $x$ rays, and shock waves. Measurements of scattered light and hard $x$ rays show a clear relation between two plasmon decay (TPD) instability and hot electrons of $T_{e}<20 \mathrm{keV}$. When the shock wave breakout was measured on the rear side of the CDF targets, shock and other preheat components prior to the shock were observed. The other components were best reproduced by a high energy tail of thermal and TPD hot electrons in a Fokker-Planck simulation.

The primary proton spectrum was measured and was shown to have a clear dependence on the compressed density of imploding pellets. The obtained $\rho R$ values are consistent with the ones measured with a conventional secondary neutron method.

Cryogenic deuterium foam with a plastic ablator and $\mathrm{CH}$ shell with $50 \mathrm{~atm} D_{2}$ gas targets were used to study the details of the implosion. The MIXS with the 10 ps time frame could show low modes structures on these shots. The core temperature was observed to drop within $50 \mathrm{ps,} \mathrm{while}$ the overall core image did not change much over a much longer time scale. $\mathrm{CH}$ shell shots with different focusing conditions show similar implosion performances in the neutron production and $x$-ray intensity, even though the MIXS images show the corresponding low mode core structures. Rayleigh-Taylor instability growth was calculated by HISHO and it was indicated that the middle range modes between 20 and 50 could be responsible for the implosion performance degradation. It is suggested that these middle modes could be reduced by introducing an ASE or partially coherent laser light into implosion experiments. 


\section{ACKNOWLEDGMENTS}

We thank the superior experimental support by the diagnostics, laser, target, and simulation groups.

${ }^{1}$ H. Takabe, K. Mima, K. Nishihara, A. Nishiguchi, M. Murakami, Y. Fukuda, H. Azechi, and S. Nakai, "Numerical studies on stability and mixing in laser driven implosion," in Proceedings of the 15th International Conference on Plasma Physics and Controlled Nuclear Fusion Research Seville, 26 September-1 October 1994 (International Atomic Energy Agency, Vienna, in press), Paper IAEA-CN-60/B-P-9.

${ }^{2}$ S. Bodner, J. Fusion Energy 1, 221 (1981).

${ }^{3}$ J. Kilkenny, S. G. Gledning, S. W. Haan, B. A. Hammel, J. D. Lindl, D. Munro, B. A. Remington, S. V. Weber, J. P. Knauer, and C. P. Verdon, Phys. Plasmas 1, 1379 (1994).

${ }^{4}$ F. J. Marshall, S. A. Letzring, C. P. Verdon, S. Skupsky, R. L. Keck, J. P. Knauer, R. L. Kremens, D. K. Bradley, T. Kessler, J. Delettrez, H. Kim, J. M. Soures, and R. L. McCrory, Phys. Rev. A 40, 2547 (1989); H. Azechi, T. Jitsuno, T. Kanabe, M. Katayama, K. Mima, N. Miyanaga, M. Nakai, S. Nakai, H. Nakaishi, M. Nakatsuka, A. Nishiguchi, P. A. Norreys, Y. Setsuhara, M. Takagi, and M. Yamanaka, Laser Part. Beams 9, 193 (1991).

${ }^{5}$ Y. Kato, K. Mima, N. Miyanaga, S. Arinaga, Y. Kitagawa, M. Nakatsuka, and C. Yamanaka, Phys. Rev. Lett. 53, 1057 (1984).

${ }^{6}$ C. Yamanaka, Y. Kato, Y. Izawa, K. Yoshida, T. Yamanaka, T. Sasaki, M. Nakatsuka, T. Mochizuki, J. Kuroda, and S. Nakai, IEEE J. Quantum Electron. QE-17, 1639 (1981).

${ }^{7}$ J. Meyer and Y. Zhu, Phys. Rev. Lett. 71, 2915 (1993).

${ }^{8} \mathrm{H}$. Shiraga, M. Heya, A. Fujishima, O. Maekawa, K. Shimada, Y. Kato, T. Yamanaka, and S. Nakai, Rev. Sci. Instrum. 66, 722 (1995).

${ }^{9}$ C. Chen, T. Norimatsu, M. Takagi, H. Katayama, T. Yamanaka, and S. Nakai, J. Vac. Sci. Technol. A 9, 340 (1991).

${ }^{10}$ M. Takagi, T. Norimatsu, T. Yamanaka, and S. Nakai, J. Vac. Sci. Technol. A 9, 820 (1991).

${ }^{11} \mathrm{M}$. Takagi, M. Ishihara, T. Norimatsu, T. Yamanaka, Y. Izawa, and S. Nakai, J. Vac. Sci. Technol. A 11, 2837 (1993).

${ }^{12}$ T. Norimatsu, H. Katayama, T. Mano, M. Takagi, R. Kodama, K. A. Tanaka, Y. Kato, T. Yamanaka, S. Nakai, Y. Nishino, M. Nakai, and C. Yamanaka, J. Vac. Sci. Technol, A 6, 3144 (1988).
${ }^{13}$ H. Katāyama; T. Norimatsu, C. Chen, T. Yamanaka, and S. Nakai, J. Vac. Sci. Technol. A 9, 2140 (1991).

${ }^{14}$ T. Norimatsu, H. Ito, C. Chen, M. Yamamoto; M. Tsukamoto, K. A. Tanaka, T. Yamanaka, and S. Nakai; Rev. Sci. Instrum. 63, 3378 (1992).

${ }^{15}$ T. A. Peyser, C. K. Manka, S. P. Obenschain, and K. J. Kearney, Phys. Fluids B 3, 1479 (1991).

${ }^{16}$ R. P. Drake, R. E. Turner, B. F. Lasinski, E. A. Williams, K. G. Estabrook, W. L. Kruer, E. M. Campbell, and T. W. Johnston, Phys. Fluids B 1, 3219 (1989).

${ }^{17}$ T. Yabe and C. Yamanaka, Comments Plasma Phys. Controlled Fusion 9, 169 (1985).

${ }^{18}$ H. Azechi, N. Miyanaga, R. O. Stapf, K. Itogawa, H. Nakaishi, M. Yamanaka, H. Shiraga, R. Tusji, S. Ido, K. Nishihara, Y. Izawa, T. Yamanaka, and C. Yamanaka, Appl. Phys. Lett. 49, 555 (1986).

${ }^{19}$ H. Furukawa and K. Nishihara, Phys. Rev. A 46, 6596 (1992).

${ }^{20}$ A. Richard, K. A. Tanaka, T. Kanabe, Y. Kitagawa, M. Nakai, K. Nishihara, T. Norimatsu, T. Yamanaka, Y. O. Fukuda, M. Katayama, M. Kado, T. Kawashima, C. Chen, M. Tsukamoto, and S. Nakai, Phys. Rev. A 49, 1520 (1994).

${ }^{21}$ W. M. Manheimer, D. G. Colombalt, and J. H. Gardner, Phys. Fluids 25, 1644 (1982); M. Kado, K. A. Tánaka, H, Yamamoto, D. W. Vick, M. Tsukamoto, N. Miyanaga, H. Azechi, A. Nishiguchi, K. Mima, and S. Nakai, J. Plasma Fusion Res. 70, 877 (1994)...

${ }^{22}$ K. Nishihara, M. Murakami, H. Azechi, T. Jitsuno, T. Kanabe, M Katayama, N. Miyanaga, M. Nakai, M. Nakatsuka, K. Tsubakimoto, and S. Nakai, Phys. Plasmas 1, 1653 (1994).

${ }^{23}$ H. Takabe, K. Mima, L. Montierth, and R. L. Morse, Phys. Fluids 28, 3676 (1985).

${ }^{24}$ H. Takabe, K. Nishihara, and T, Taniuchi, J. Phys, Soc. Jpn. 45, 2001 (1978).

${ }^{25}$ S. T. Haan, Phys. Rev. A 39, 5812 (1989).

${ }^{26} \mathrm{H}$. Nakano, K. Tsubakimoto, N. Miyanaga, M. Nakatsuka, T. Kanabe, Azechi, T. Jitsuno, and S. Nakai, J. Appl. Phys. 73, 2122 (1993); H. Nakano, N. Miyanaga, K. Yagi, K. Tsubakimoto, T. Kanabe, M. Nakatsuka, and S. Nakai, Appl. Phys. Lett. 63, 580 (1993). 